\title{
Pattern of Health-Related Quality of Life and its Association among Patients with Colorectal Cancer
}

\author{
Bello Arkilla Magaji ${ }^{1,2}$, Foong Ming Moy ${ }^{2}$, Chee Wei Law ${ }^{3}$, Hoe Leong Sii ${ }^{4}$, April \\ Camilla Roslani ${ }^{3}$
}

${ }^{1}$ Department of Community Health, Faculty of Clinical Sciences, College of Health Sciences, Usmanu Danfodiyo University, PMB 2346, Sokoto Nigeria. ${ }^{2}$ Department of Social \& Preventive Medicine, Faculty of Medicine, University of Malaya, 50603 Kuala Lumpur, Malaysia. ${ }^{3}$ Department of Surgery, Faculty of Medicine, University of Malaya, 50603 Kuala Lumpur, Malaysia. ${ }^{4}$ Faculty of Medicine, University of Malaya, 50603 Kuala Lumpur, Malaysia.

\begin{abstract}
Aim: Research on patient-reported outcomes in colorectal cancer are scarce in Malaysia. We aimed to determine pattern of health-related quality of life and its associations among patients with colorectal cancer. Methods: A cross-sectional study of 324 patients. Data were drawn from patient's records and interviews. Research tools included the locally validated European Organization for Research and Treatment of Cancer (EORTC) core QLQ-C30 and colorectal-cancer specific QLQ-CR29 questionnaires. Results: The mean ( \pm standard deviation) age of patients was $62.5( \pm 13.6)$ years. Majority of them were Chinese $(62.3 \%)$ followed by Malays $(19.8 \%)$, Indians (14.8\%) and others (3.1\%). Colon cancer was 7 -fold more common than rectal cancer (87\% vs $13 \%)$. Majority of them were presented at Stage II (29.6\%) and III (28.1) of TNM Staging. The mean ( \pm SD) score for global health status/quality of life (GHS/QOL) was $73.13( \pm 14.19)$. Good overall functionality (mean scores $\geq 80$ ) was observed for physical, emotional, role, cognitive and social/family for QLQ-C30; body image, anxiety and weight for QLQ-CR29. The mean score for sexual functioning in men (32.95 \pm 28.81$)$ and women (17.56 \pm 25.60$)$ were low. Commonest symptoms were fatigue (22.29 \pm 17.40$)$, urinary frequency $(21.96 \pm 24.22)$ and insomnia $(18.42 \pm 23.32)$. Those with lower GHS/QOL had stoma $(\mathrm{p}=0.044)$ and were of Chinese ethnicity $(\mathrm{p}<0.001)$. On multivariate analysis, ethnicity was the only independent predictor of GHS/QOL ( $95 \%$ confidence interval, 2.589 to $6.139, \mathrm{p}<0.001)$. Conclusion: Our results suggest that there is decrease in sexual functioning despite good overall GHS/QOL among our colorectal cancer survivors. Fatigue, sleep loss and urinary frequency were the common symptoms after cancer treatment. Chinese patients had the poorest quality of life.
\end{abstract}

Keywords: Quality of life- colorectal cancer- EORTC QLQ-C30- EORTC QLQ-CR29- global health status/quality of life

Asian Pac J Cancer Care, 4 (2), 45-52

\section{Introduction}

Health-related quality of life (HRQOL) of colorectal cancer survivors is a growing public health concern, alongside conventional outcome measures that are disease-centric such as mortality and survival rate. Despite being the third most common cancer and the fourth leading cause of cancer-related deaths worldwide, the survival rate of colorectal cancer continues to increase substantially over the past decades [1]. The 5-year survival rates for both colon and rectal cancer have been reported to increase by at least $30 \%$ respectively over a
Submission Date: 12/11/2018 Acceptance Date: 02/08/2019

40-year duration [2]. Besides, colorectal cancer had the largest gain in surviving outcomes among several cancers studied from a population-based registries in Australia, Canada, Denmark, Norway, Swedish regions and UK where the survival rates have increased at a similar pace in these regions [3].

In Malaysia, colorectal cancer is the second leading cancer, accounts for $13.2 \%$ of all cancers [4]. It is now the most common cancer that replaced lung cancer in male and the second most common in female, comprising $14.6 \%$ and $11.1 \%$ of male and female cancers respectively. [4]. Comparing the three major ethic groups in Malaysia,

Corresponding Author:

Dr. Foong Ming Moy

Department of Social \& Preventive Medicine, Faculty of Medicine, University of Malaya, 50603 Kuala Lumpur, Malaysia.

Email: moyfm@ummc.edu.my 
those of Chinese ethnicity had the highest incidence (age-standardised rate of 21.1 per 100,000) as compared to Malays (11.3 per 100,000) and Indians (10.3 per 100,000). Local studies reported that up to $76 \%$ and $60 \%$ of patients with colorectal cancer survived through the first and five years after diagnosis respectively [5-6]. As the long-term survival rate has improved over different regions, colorectal cancer survivors are therefore having higher expectations to optimize the quality on their well-being during the post-treatment period [7-8].

Understanding the pattern of HRQOL as well as the complexity of factors, characteristics or conditions that predict HRQOL is a significant step towards identifying patients who are at risk of poor HRQOL. Previous studies that have investigated the role of socio-demographic, medical, lifestyle behaviour, health system and various intervention in predicting the HRQOL of colorectal cancer patients have been inconsistent [9-12]. In addition, there are limited studies assessing the functionality and symptoms of colorectal cancer using the newest European Organization for Research and Treatment of Cancer (EORTC) Colorectal Cancer-specific Quality of Life Questionnaire (QLQ-CR29). Targeted interventions could be done effectively on potentially modifiable factors that are predictive of poorer HRQOL [9-11].

Therefore, we embarked on this study to assess patient's perceived global health status and quality of life (GHS/QOL), functional outcomes and symptoms as measured by the Malaysian versions of Quality of Life Questionnaire core (QLQ-C30) and QLQ-CR29; and to determine factors among demographics, clinical factors, treatment and scales of the QLQ-C30/ QLQ-CR29 that were associated with of GHS/QOL in patients with colorectal cancer.

\section{Materials and Methods}

\section{Study design and subjects}

This was a cross-sectional study conducted at the University Malaya Medical Centre (UMMC), which is a tertiary hospital. Ethics approval was obtained from the Medical Research Ethics Committee (MREC ID: PPUM/ UPP/300/02/02, MEC 770.2). Colorectal cancer survivors were sampled via the Electronic Medical Record (EMR) system from the medical centre. Patients were eligible for the study if 1) they were Malaysian citizens or permanent residents, 2) aged 18 years and above at the time of diagnosis, 3) diagnosis were primary cancer of colon/ rectum, and 4) they received at least one form of treatment (surgery, chemotherapy or radiotherapy). Those with mental impairment or mental illness and those unable to read or understand any of the two languages (Malaysian national language, Malaysian Chinese) were excluded from the study. The protocol of this study were validated and published elsewhere [13]. Informed consent was obtained from each patient before data collection.

\section{Sample size}

Sample size was calculated based on previous literature by Neil W Scott et al., considering Malaysian colorectal patients had global health mean score of 10 points higher than the reference values of 60.7 (SD 23.4) as reported in the literature [14]. The formula in the reference manuals of the EORTC QLQ-C30 was used. P-value of $<0.05$ was considered as significant and power was considered as $80 \%$. To achieve this, 174 patients were needed to enrol into the study.

\section{Data collection}

Data collection was conducted between May and June 2013. Information on baseline socio-demographic and medical conditions, index cancer clinical features, treatment characteristic and the consequences of the disease and its treatments were obtained from the EMR system. While the functional scales, symptoms and single item scales of the EORTC QLQ-C30 and QLQ-CR29 questionnaires were obtained through the phone interviews. A total of four experienced research assistants were recruited, two for Malaysian national language and two for Malaysian Chinese language. During the phone interviews, patients were given detailed explanation on the purpose and methods of study, the right to participate or refuse and the language of preference for the questionnaire. Patients were requested to respond to the questionnaire themselves with no proxies allowed.

\section{Study instruments}

HRQOL scales were measured using locally validated questionnaire of the EORTC QLQ-C30 and CR-29. Validation of EORTC QLQ-C30 and QLQ-CR29 were necessary due to different cultural settings and languages in Malaysia. This has been translated into Malaysian national language [15-17] and Malaysian Chinese [18] and were validated.

The QLQ-C30 is one of the most widely used questionnaire in clinical cancer research [19]. It comprises of 30 items, representing five functioning sub-scales, three symptoms scales, GHS/QOL scale, six single items. The QLQ-CR29 is the newest colorectal cancer specific module comprising 29 items [20]. The questionnaire was administered with QLQ-C30. It consists of one multi-item function scale, four single items assessing functioning, three symptoms scale, single item symptoms and sexual pleasure. High score in functioning scales represents better level of functioning whereas high score in symptom scales represents worse or severe symptoms.

\section{Data analysis}

All statistical analyses were conducted using SPSS version 22.0 for Windows (SPSS Inc., and Chicago, Illinois, USA). Exploratory analysis was performed to examine the accuracy and completeness of our data as well as to guide the choice of statistical methods. Raw scores from the QLQ-C30 and QLQ-CR29 were transformed into a scale of 0-100 according to the developer's guidelines. The profile of patients' HRQOL was presented using mean and standard deviation $( \pm \mathrm{SD})$

Univariate analysis was performed to compare the difference in patients' GHS/QOL, functionality and symptoms experience according to patients' 
characteristics, comorbidities and treatment modalities. Independent sample t-test and one-way analysis of variance (ANOVA) with Bonferroni post-hoc tests of contrasts were used. Chi-square test was used to compare proportions between categorical variables. Pearson correlations coefficient, Kruskal Wallis tests were used to compare mean in different HRQOL scales and correlation coefficient ( $r$ ) with corresponding p-value were presented accordingly. Variables with $\mathrm{p}$ values of less than 0.25 were included in the multivariate analysis. Stepwise multiple linear regression analysis (3 levels) modelling was conducted with all the potential predictors and the outcome variable.

\section{Results}

A total of 324 patients were recruited and included in the final analysis. Majority of them were of Chinese ethnicity (62.3\%), followed by Malays (19.8\%) and Indians $(14.8 \%)$. They had a mean age $( \pm \mathrm{SD})$ of 62.5 years $( \pm 13.6)$, with a mean duration of 62.8 months $( \pm 31.4)$ since diagnosis. Overall, $87 \%$ of our participants were diagnosed with colon cancer, up to 7-fold commoner than rectal cancer (13\%). TNM staging at presentation to the hospital was as follows: Stage I (7.1\%), Stage II (29.6\%), Stage III (28.1\%) and Stage IV (16.0\%), while the remaining $19.1 \%$ was unknown. The most common treatment modality was surgery alone $(42.9 \%)$. Stoma was present in about $10 \%$ of patients. The baseline characteristic of the overall patient cohort is shown in Table 1.

\section{Health-related quality of life (HRQOL) in colorectal cancer patients}

In general, majority of patients rated their HRQOL measures as high. About $89 \%$ of patients and $87 \%$ of patients rated their global health status (GHS) and overall quality of life (QOL) between score of 5 and 7 respectively on a scale of 1 to 7 (from 'very poor' to 'excellent'). After linear transformation from raw scores to a scale of 0-100, the mean score for GHS/QOL was 73.23.

A better functioning is represented by a higher score in functioning scales. With respect to the QLQ-C30 core module, high functioning abilities were observed in our patients with mean scores of above 80 in all aspects of functionality. Highest functioning score was reported for emotional functioning $($ mean $=88.17)$ whereas role functioning $($ mean $=83.02)$ scored the lowest. On the contrary, higher score in symptom scales represents a common/ worst symptom. Fatigue (mean=22.29) was the most common/worst symptom, followed by sleep loss (mean 18.42), constipation $($ mean $=15.02)$ and pain $(13.79)$. Financial difficulty $($ mean $=11.32)$ was perceived as relatively modest problem among colorectal cancer patients in term of lack of job and care-related expenditure.

Most patients were satisfied with their body image $($ mean $=94.27)$ as it scored the highest among the functioning scales covered in colorectal cancer specific QLQ-CR29 module. However, mean scores for sexual dysfunction in both men (mean= 33.14) and women $($ mean $=17.56)$ were remarkably low, indicating significant sexual dysfunction in colorectal cancer patients. The most common/ worst symptoms specific to colorectal cancer reported by patients were urinary frequency $($ mean $=21.96)$, stoma care related problem (in patients with stoma) $($ mean $=20.00)$, impotence (in men) (mean= 18.49) and flatulence (mean= 16.87). Table 2 presents the mean scores and standard deviations for the GHS/QOL, functioning scales and symptoms experience.

\section{Factors associated with HRQOL}

On univariate analysis, presence of stoma and ethnicity were significantly associated with health-related quality of life among colorectal cancer patients. The mean score of GHS/QOL is significantly lower in Chinese (mean=70.13) than Malays (mean=77.73), Indians (mean= 78.65) and others (mean=80.83). Besides, there was significantly higher mean score observed among patients with stoma $($ mean $=73.63)$ than those without stoma $($ mean= 69.35). Results are shown in Table 1.

Variables with p-value of less than 0.25 were included in multivariate analysis. Factors included were gender, ethnicity, duration since diagnosis, hypertension, presence of stoma and treatment modality. Of these, ethnicity was the only independent predictor of GHS/ QOL (95\% confidence interval, 2.589 to 6.139, $\mathrm{p}<0.001$ ). Further post-hoc analysis revealed that Indians and Malays had significantly higher scores compared to Chinese. Age, gender, body mass index (BMI), duration of diagnosis, comorbidities, anatomic site of tumour, TNM's staging, treatment modality and presence of stoma were not associated with GHS/QOL. Results are shown in Table 3.

We further stratified the dimensions of HRQOL by the ethnicity. It was found that all the domains including functionalities and symptoms in QLQ-C30 were significantly associated with different ethnic groups whereas some domains in QLQ-CR29 were significantly associated with ethnic groups. Chinese had significantly poorer GHS/QOL in all domains of QLQ-C30 core module as compared to Malays, Indians and others. Besides, Chinese had poorer functionalities (excluding sexual dysfunction in men) and experienced worst symptoms such as urinary frequency, bloated feeling, dry mouth, trouble with taste, hair loss and stoma care related problems than other ethnic groups according to the QLQ-CR29 module.

\section{Discussion}

Colorectal cancer and its temporal development in developing countries including Malaysia remains a significant health burden and public health concern. With increased post-treatment survival rate, research on the pattern of quality of life and its associated factors is crucial to guide physicians in choosing treatment options and to improve quality of life by giving proper intervention on modifiable factors [9-11]. In this study, we evaluated quality of life of colorectal cancer patients who received 
Table 1. Baseline Characteristic and Its Association with Global Health Status/quality of Life (GHS/QOL) of Colorectal Cancer Patients. Total $(n=324)$

\begin{tabular}{|c|c|c|c|}
\hline Characteristics & Number (Percentage) & Overall GHS/QOL * & $\mathrm{p}$ value \\
\hline Age at diagnosis (years) $*$ & $62.45( \pm 13.64)$ & & \\
\hline Age at diagnosis (years) & & & 0.437 \\
\hline$\leq 60$ & $131(40.4)$ & $73.79( \pm 15.09)$ & \\
\hline$\geq 61$ & $193(59.6)$ & $72.80( \pm 13.51)$ & \\
\hline Gender & & & 0.198 \\
\hline Male & $163(50.3)$ & $73.16( \pm 14.64)$ & \\
\hline Female & $161(49.7)$ & $73.29( \pm 13.76)$ & \\
\hline Body mass index $\left(\mathrm{kg} / \mathrm{m}^{2}\right)^{*}$ & $22.35( \pm 4.99)$ & & \\
\hline Body mass index $\left(\mathrm{kg} / \mathrm{m}^{2}\right)$ & & & 0.427 \\
\hline Normal weight $(<25)$ & $140(43.2)$ & $72.32( \pm 14.87)$ & \\
\hline Overweight (25-30) & $176(54.3)$ & $74.10( \pm 13.70)$ & \\
\hline Obese $(>30)$ & $8(2.5)$ & $69.79( \pm 12.55)$ & \\
\hline Ethnicity & & & $<0.001$ \\
\hline Chinese & $202(62.3)$ & $70.13( \pm 14.14)$ & \\
\hline Malays & $63(19.8)$ & $77.73( \pm 10.61)$ & \\
\hline Indians & $48(14.8)$ & $78.65( \pm 15.56)$ & \\
\hline Others & $10(3.1)$ & $80.83( \pm 11.15)$ & \\
\hline Duration since diagnosis (months) * & $62.78( \pm 31.36)$ & & \\
\hline Duration since diagnosis (months) & & & 0.053 \\
\hline$\leq 60$ & $158(48.5)$ & $73.26( \pm 14.45)$ & \\
\hline$\geq 61$ & $166(51.5)$ & $73.19( \pm 13.98)$ & \\
\hline \multicolumn{4}{|l|}{ Comorbidities } \\
\hline Hypertension & & & 0.234 \\
\hline Yes & $86(26.5)$ & $73.06( \pm 13.33)$ & \\
\hline No & $238(73.5)$ & $73.28( \pm 14.51)$ & \\
\hline Diabetes & & & 0.753 \\
\hline Yes & $51(15.7)$ & $73.86( \pm 13.02)$ & \\
\hline No & $273(84.3)$ & $73.11( \pm 14.42)$ & \\
\hline Anatomic site of tumour & & & 0.713 \\
\hline Colon & $282(87.0)$ & $73.20( \pm 14.39)$ & \\
\hline Rectum & $42(13.0)$ & $73.41( \pm 12.91)$ & \\
\hline TNM Staging & & & 0.746 \\
\hline I & $23(7.1)$ & $73.55( \pm 11.95)$ & \\
\hline II & $96(29.6)$ & $72.92( \pm 14.20)$ & \\
\hline III & $91(28.1)$ & $74.54( \pm 14.18)$ & \\
\hline $\mathrm{V}$ & $52(16.0)$ & $71.15( \pm 12.18)$ & \\
\hline Unknown & $62(19.1)$ & $73.39( \pm 15.52)$ & \\
\hline Treatment & & & 0.085 \\
\hline Surgery alone & $139(42.9)$ & $73.02( \pm 12.91)$ & \\
\hline Surgery and chemo or radiotherapy & $123(38.0)$ & $74.33( \pm 15.37)$ & \\
\hline Surgery and chemo and radiotherapy & $10(3.1)$ & $78.33( \pm 11.25)$ & \\
\hline Chemo and/or radiotherapy & $20(6.2)$ & $75.00( \pm 11.15)$ & \\
\hline Palliative & $32(9.9)$ & $67.19( \pm 16.11)$ & \\
\hline Presence of stoma & & & 0.044 \\
\hline Yes & $31(9.6)$ & $69.35( \pm 13.16)$ & \\
\hline No & $293(90.4)$ & $73.63( \pm 14.25)$ & \\
\hline
\end{tabular}

Abbreviation, GHS/QOL, global health status/quality of life; * Values are expressed as mean ( \pm standard deviation) 
Table 2. Overall Scores for the Global Health Status/ Quality of Life (GHS/QOL), Functioning Scales and Symptoms Experience

\begin{tabular}{|c|c|}
\hline Variables & Scores * \\
\hline \multicolumn{2}{|l|}{ EORTC QLQ-C30 module } \\
\hline Overall GHS/QOL & $73.23( \pm 14.19)$ \\
\hline \multicolumn{2}{|l|}{ Functioning scales } \\
\hline Physical & $84.34( \pm 16.58)$ \\
\hline Emotional & $88.17( \pm 17.32)$ \\
\hline Role & $83.02( \pm 20.23)$ \\
\hline Cognitive & $83.90( \pm 19.99)$ \\
\hline Social/ family & $88.01( \pm 18.30)$ \\
\hline \multicolumn{2}{|l|}{ Multi-item symptom scales } \\
\hline Fatigue & $22.29( \pm 17.40)$ \\
\hline Nausea/ vomiting & $7.30( \pm 13.43)$ \\
\hline Pain & $13.79( \pm 18.23)$ \\
\hline \multicolumn{2}{|l|}{ Single item symptom scales } \\
\hline Dyspnoea & $10.70( \pm 18.79)$ \\
\hline Sleep loss & $18.42( \pm 23.32)$ \\
\hline Appetite loss & $13.07( \pm 20.08)$ \\
\hline Constipation & $15.02( \pm 22.43)$ \\
\hline Diarrhoea & $11.01( \pm 17.95)$ \\
\hline Financial difficulty & $11.32( \pm 17.26)$ \\
\hline \multicolumn{2}{|l|}{ EORTC QLQ-CR29 module } \\
\hline \multicolumn{2}{|l|}{ Functional scales } \\
\hline Body image & $94.27( \pm 12.59)$ \\
\hline Anxiety & $82.41( \pm 20.38)$ \\
\hline Weight & $91.05( \pm 16.76)$ \\
\hline Sexual dysfunction in men & $33.14( \pm 28.87)$ \\
\hline Sexual dysfunction in women & $17.56( \pm 25.59)$ \\
\hline \multicolumn{2}{|l|}{ Multi-item symptom scales } \\
\hline Urinary frequency & $22.96( \pm 24.22)$ \\
\hline Blood and mucus & $1.03( \pm 5.92)$ \\
\hline Stool frequency & $5.14( \pm 15.04)$ \\
\hline \multicolumn{2}{|l|}{ Single item symptom scales } \\
\hline Urinary incontinence & $4.63( \pm 13.97)$ \\
\hline Dysuria & $2.98( \pm 11.49)$ \\
\hline Abdominal pain & $4.63( \pm 13.21)$ \\
\hline Buttock pain & $1.85( \pm 8.89)$ \\
\hline Bloated feeling & $10.49( \pm 17.58)$ \\
\hline Dry mouth & $13.37( \pm 20.13)$ \\
\hline Hair loss & $14.19( \pm 23.96)$ \\
\hline Trouble with taste & $7.61( \pm 15.19)$ \\
\hline Flatulence & $16.87( \pm 21.71)$ \\
\hline Faecal incontinence & $3.60( \pm 14.03)$ \\
\hline Sore skin & $2.78( \pm 12.68)$ \\
\hline Embarrassment & $2.37( \pm 12.21)$ \\
\hline Stoma care-related problem & $20.00( \pm 31.91)$ \\
\hline Impotence & $18.49( \pm 26.74)$ \\
\hline Dyspareunia & $3.82( \pm 12.15)$ \\
\hline
\end{tabular}

Abbreviation, GHS/QOL, global health status/quality of life; EORTC, European Organisation for Research and Treatment of Cancer; * Values are expressed in mean ( \pm standard deviation) treatments in UMMC and to determine factors associated with quality of life.

The demographic, clinical and treatment characteristics of our patients were generally similar to those reported by previous studies from Malaysia and other countries in the South East Asian region [21-24]. The mean age of our participants was found to be around 62 years and up to $60 \%$ were of Chinese ethnicity. The number of male and female patients was almost equal. Besides, majority of patients included in this study presented with advanced stage cancer in which up to $45 \%$ were staged TNM's Stage III and IV. This could be due to the fact that most patients diagnosed with advanced cancer were usually referred to tertiary hospital such as our hospital.

Health-related quality of life among our patients was good with respect to the global health status and overall quality of life (GHS/QOL) with an overall mean score of 73. This is comparable with previous studies in which the reported mean score for global quality of life ranged from 60 to 75 [12, 25-30], and confirmed the findings from a smaller preliminary study in local cohort [31]. Apart from that, our patients reported relatively high scores (more than 80) for functionalities that include physical, emotional, role, cognitive, social/family for QLQ-C30 as well as body image, anxiety and body weight for QLQ-CR29. This could be explained by the better colorectal cancer management at our tertiary centre that involve multidisciplinary team such as colorectal surgeons, oncologists, palliative physicians and pain management team. Good overall functionalities were also reported elsewhere for QLQ-C30 [25-29] and QLQ-CR29 [30-32]. However, based on previous literature, there were substantial deficits observed for functioning in patients with colorectal cancer survivors as compared with normal population [27-33]. As we did not include normal population, we were not able to compare the difference in functioning between these two groups.

Of note, sexual dysfunction is one of the main concern among our patients with low mean scores of 33 in men and 17 in women respectively. Similar results were demonstrated in which the post-treatment sexual functioning scores were low, ranged from 15 to 40 [25-34, 35]. This confirms that sexual functioning is an essential component of quality of life that should be addressed adequately as it is one of the most common long-term effects of cancer treatment [36]. The impact of colorectal cancer treatments on sexual functioning is multifactorial and could be attributed to surgical scars, presence of stoma, urinary/faecal incontinence, fatigue and erectile dysfunction [36]. Patients often feel too embarrassed to raise sex-related health issues to healthcare providers [37]. Educating patients and providing them with treatment options could help to improve sexual functioning in colorectal cancer survivors [38].

Despite good overall GHS/QOL and functionalities, some symptoms seem to persist among our patients. We found that our patients suffered from long-lasting physical symptoms such as fatigue and sleep loss, genitourinary symptoms such as urinary frequency as well as bowel symptoms such as constipation. Several 
Table 3. Association between Global Health Status/quality of Life (GHS/QOL) and Baseline Characteristic of Patients

\begin{tabular}{lccccc}
\hline Dependent variable & Covariates & \multicolumn{2}{c}{ Regression coefficient, R } & \multicolumn{2}{c}{$95 \%$ confidence interval } \\
\hline \multirow{2}{*}{ GHS/QOL } & Gender & B (SE) & p value & Lower bound & Upper bound \\
& Ethnicity & $4.241(.907)$ & $<0.001$ & -2.836 & 3.181 \\
& Duration since diagnosis & $.343(1.635)$ & 0.834 & 2.456 & 6.025 \\
& Hypertension & $-.372(1.750)$ & 0.832 & -2.874 & 3.561 \\
& Presence of stoma & $-3.372(2.759)$ & 0.222 & -8.800 & 3.071 \\
& Treatment modality & $-.641(.611)$ & 0.295 & -1.844 & 0.561 \\
\hline
\end{tabular}

Abbreviation, GHS/QOL, global health status/quality of life; SE, standard error

studies also reported persistent physical symptoms in colorectal cancer survivors such as reduction in energy [26-39], genitourinary symptoms such as frequency and incontinence [35] and bowel problem including constipation and diarrhoea [29-40]. Not only that, psychological distress and depression have been found to be a significant problem among long-term colorectal cancer survivors even after they achieved remission for years [41].

Presence of stoma is likely to impair patients' quality of life. The association between stoma and poorer quality of life have been studied and was further demonstrated when the quality of life improved consistently after reversal of stoma [42]. Patients with stoma have been shown to have limited functioning in their daily activities related to work and hobbies. Apart from that, they would feel less satisfied with body image and this further impaired their family and social functioning [28]. In this study, the mean score for GHS/QOL in patients with stoma is lower than those without stoma (69 vs 74) but it was not statistically significant on multivariate analysis. This might be due to the small number $(10 \%)$ of our patients who had stoma during recruitment.

To our surprise, ethnicity is the only independent predictor of health-related quality of life among colorectal cancer survivors. No association between ethnicity and quality of life were reported previously except that there is one study reported a trend for higher psychological quality of life among African American survivors than Caucasion survivors [43]. We found that Chinese had the poorest quality of life in comparison with Malays and Indians. After stratifying the QLQ-C30 and QLQ-CR29 module according to different ethnic groups, Chinese was found to have poorer quality of life in all domains in functioning as well as symptoms scales that were examined in QLQ-C30 core module. Besides, some domains in the QLQ-CR29 were significantly more impaired in Chinese patients than Malay and Indian patients. The reason for this observation might be the cultural factors and traditional beliefs among different ethnic groups that possess different perceptions towards quality of life. Socio-cultural factors such as education, income, family/social support might play a role in determining the perception of quality of life.

This study demonstrated that the quality of life among our colorectal cancer survivors is comparable with other studies. However, there were several limitations that should be addressed while interpreting our results. Firstly, there may be sampling bias as the data were obtained from only one institution and therefore may not be generalised to the general population of colorectal cancer patients. Besides, as half of our patients survived more than five years, this may not reflect the situation for those who died before the five-year follow-up. On the other hand, the strength of our study includes the use of well-established instruments to assess HRQOL among colorectal cancer survivors, in which comparisons can be made with studies from both local and international settings. We also highlighted that despite good overall GHS/QOL, sexual dysfunction is prevalent among Malaysian patients which need to be addressed in future.

In conclusion, our results suggest that there is decrease in sexual functioning despite good overall GHS/QOL among our colorectal cancer survivors. Fatigue, sleep loss, urinary frequency and constipation were the common symptoms after cancer treatment. Chinese had poorer quality of life among colorectal cancer survivors.

\section{Acknowledgments}

The authors thank the patients, research assistants, and members of the colorectal surgery units of the UMMC for the assistance they rendered towards the success of this study. We thank the staff of the European Organization for Research and Treatment of Cancer Quality of Life Department, Avenue Mounier, 83/11 1200 Brussels, Belgium for the support offered to us. We also wish to acknowledge the funders of this study. This study was fully funded by the University of Malaya postgraduate fund with the following reference numbers (UM-IPPP PS212/2010A and PV015-2011B) and partially supported by the STeMM Programme, the University of Malaya/Ministry of Higher Education (UM/MOHE) High Impact Research Grant (No: E000010-20001).

\section{Conflict of interest}

The authors declare no conflict of interest.

\section{References}

1. Arnold M, Sierra MS, Laversanne M, Soerjomataram I, Jemal A, Bray F. Global patterns and trends in colorectal cancer incidence and mortality. Gut. 2017;66(4):683-91.

2. Quaresma M, Coleman MP, Rachet B. 40-year trends in an index of survival for all cancers combined and survival adjusted for age and sex for each cancer in England and 
Wales, 1971-2011: a population-based study. The lancet. 2015;385(9974):1206-18.

3. Coleman M, Forman D, Bryant H, Butler J, Rachet B, Maringe C, et al. Cancer survival in Australia, Canada, Denmark, Norway, Sweden, and the UK, 1995-2007 (the International Cancer Benchmarking Partnership): an analysis of population-based cancer registry data. The Lancet. 2011;377(9760):127-38

4. Azizah Ab M NSIT, Noor Hashimah A, Asmah Z A, Mastulu W. Malaysian National Cancer Registry Report 2007-2011. 2016.

5. Kong C-K, Roslani AC, Law C-W, Law S, Arumugam K. Impact of socio-economic class on colorectal cancer patient outcomes in Kuala Lumpur and Kuching, Malaysia. Asian Pac J Cancer Prev. 2010;11(4):969-74.

6. Magaji BA, Moy FM, Roslani AC, Law CW. Survival rates and predictors of survival among colorectal cancer patients in a Malaysian tertiary hospital. BMC cancer. 2017;17(1):339.

7. Hudson MM, Landier W, Ganz PA. Impact of survivorshipbased research on defining clinical care guidelines. Cancer Epidemiology and Prevention Biomarkers. 2011;20(10):2085-92.

8. Baade PD, Youlden DR, Chambers SK. When do I know I am cured? Using conditional estimates to provide better information about cancer survival prospects. Medical Journal of Australia. 2011;194(2):73.

9. Schlesinger S, Walter J, Hampe J, von Schönfels W, Hinz S, Küchler T, et al. Lifestyle factors and health-related quality of life in colorectal cancer survivors. Cancer Causes \& Control. 2014;25(1):99-110.

10. Steginga SK, Lynch BM, Hawkes A, Dunn J, Aitken J. Antecedents of domain-specific quality of life after colorectal cancer. Psycho-Oncology: Journal of the Psychological, Social and Behavioral Dimensions of Cancer. 2009;18(2):216-20.

11. Yost KJ, Hahn EA, Zaslavsky AM, Ayanian JZ, West DW. Predictors of health-related quality of life in patients with colorectal cancer. Health and Quality of Life Outcomes. 2008;6(1):66.

12. Chambers SK, Meng X, Youl P, Aitken J, Dunn J, Baade P. A five-year prospective study of quality of life after colorectal cancer. Quality of Life Research. 2012;21(9):1551-64.

13. Magaji BA, Moy FM, Roslani AC, Sagap I, Zakaria J, Blazeby JM, et al. Health-related quality of life among colorectal cancer patients in Malaysia: a study protocol. BMC cancer. 2012;12(1):384.

14. Scott NW, Fayers P, Aaronson NK, Bottomley A, de Graeff A, Groenvold M, et al. EORTC QLQ-C30 reference values manual. 2008.

15. Magaji BA MF, Roslani AC, Wei C, Law FR. . Psychometric Validation of the Bahasa Malaysia Version of the EORTC QLQ-CR29. Asian Pacific Journal of Cancer Prevention. 2015;16(18):8101-5.

16. Na Y, WYb L, CHc Y. The Malay version of the European Organization for Research and Treatment of Cancer Quality of Life Questionnaire (EORTC-QLQ C30): reliability and validity study. Int Med J Malaysia. 2010.

17. Magaji BA, Moy FM, Roslani AC, Law CW, Raduan F, Sagap I. Psychometric Validation of the Bahasa Malaysia Version of the EORTC QLQ-CR29. Asian Pacific journal of cancer prevention : APJCP. 2016;16(18):8101-5.

18. Magaji BA, Moy FM, Roslani AC, Law CW, Sagap I. Psychometric validation of the Malaysian Chinese version of the EORTC QLQ-C30 in colorectal cancer patients. Asian Pac J Cancer Prev. 2015;16(18):8107-12.

19. Aaronson NK, Ahmedzai S, Bergman B, Bullinger M, Cull A, Duez NJ, et al. The European Organization for Research and
Treatment of Cancer QLQ-C30: a quality-of-life instrument for use in international clinical trials in oncology. JNCI: Journal of the National Cancer Institute. 1993;85(5):365-76.

20. Whistance R, Conroy T, Chie W, Costantini A, Sezer O, Koller M, et al. Clinical and psychometric validation of the EORTC QLQ-CR29 questionnaire module to assess healthrelated quality of life in patients with colorectal cancer. European journal of cancer. 2009;45(17):3017-26.

21. Chong V, Abdullah M, Telisinghe P, Jalihal A. Colorectal cancer: incidence and trend in Brunei Darussalam. Singapore medical journal. 2009;50(11):1085.

22. GOH KL, QUEK KF, Yeo G, Hilmi I, LEE CK, Hasnida $\mathrm{N}$, et al. Colorectal cancer in Asians: a demographic and anatomic survey in Malaysian patients undergoing colonoscopy. Alimentary pharmacology \& therapeutics. 2005;22(9):859-64.

23. R. Hassan M, Khamizar W, Raihan N, K. Kiew K. Incidence of colorectal cancer in Kedah and Perlis, Malaysia2010. A73-A p.

24. Magaji BA, Moy FM, Roslani AC, Law CW. Descriptive epidemiology of colorectal cancer in University Malaya Medical Centre, 2001 to 2010. Asian Pac J Cancer Prev. 2014;15(15):6059-64.

25. Allal AS, Gervaz P, Gertsch P, Bernier J, Roth AD, Morel $\mathrm{P}$, et al. Assessment of quality of life in patients with rectal cancer treated by preoperative radiotherapy: a longitudinal prospective study. International Journal of Radiation Oncology* Biology* Physics. 2005;61(4):1129-35.

26. Arndt V, Merx H, Stegmaier C, Ziegler H, Brenner H. Quality of life in patients with colorectal cancer 1 year after diagnosis compared with the general population: a population-based study. Journal of Clinical Oncology. 2004;22(23):4829-36.

27. Arndt V, Merx H, Stegmaier C, Ziegler H, Brenner H. Restrictions in quality of life in colorectal cancer patients over three years after diagnosis: a population based study. European Journal of Cancer. 2006;42(12):1848-57.

28. Engel J, Kerr J, Schlesinger-Raab A, Eckel R, Sauer H, Hölzel D. Quality of life in rectal cancer patients: a fouryear prospective study. Annals of surgery. 2003;238(2):203.

29. Pucciarelli S, Del Bianco P, Toppan P, Serpentini S, Efficace F, Pasetto LM, et al. Health-related quality of life outcomes in disease-free survivors of mid-low rectal cancer after curative surgery. Annals of surgical oncology. 2008;15(7):1846-54.

30. Peng J, Shi D, Goodman KA, Goldstein D, Xiao C, Guan $\mathrm{Z}$, et al. Early results of quality of life for curatively treated rectal cancers in Chinese patients with EORTC QLQ-CR29. Radiation Oncology. 2011;6(1):93.

31. Natrah M, Ezat SW, Syed M, Rizal A, Saperi S. Quality of life in Malaysian colorectal cancer patients: a preliminary result. Asian Pacific Journal of Cancer Prevention. 2012;13(3):957-62.

32. Wiltink LM, Chen TY, Nout RA, Kranenbarg EM-K, Fiocco M, Laurberg S, et al. Health-related quality of life 14 years after preoperative short-term radiotherapy and total mesorectal excision for rectal cancer: report of a multicenter randomised trial. European journal of cancer. 2014;50(14):2390-8.

33. Bentzen AG, Balteskard L, Wanderås EH, Frykholm G, Wilsgaard T, Dahl O, et al. Impaired health-related quality of life after chemoradiotherapy for anal cancer: late effects in a national cohort of 128 survivors. Acta Oncologica. 2013;52(4):736-44.

34. Camilleri-Brennan J, Steele R. Prospective analysis of quality of life and survival following mesorectal excision for rectal cancer. British Journal of Surgery. 2001;88(12):1617-22.

35. Herman JM, Narang AK, Griffith KA, Zalupski MM, Reese JB, Gearhart SL, et al. The quality-of-life effects 
of neoadjuvant chemoradiation in locally advanced rectal cancer. International Journal of Radiation Oncology* Biology* Physics. 2013;85(1):e15-e9.

36. Averyt JC, Nishimoto PW. Addressing sexual dysfunction in colorectal cancer survivorship care. Journal of gastrointestinal oncology. 2014;5(5):388.

37. Althof SE, Parish SJ. Clinical interviewing techniques and sexuality questionnaires for male and female cancer patients. The journal of sexual medicine. 2013;10:35-42.

38. Traa M, De Vries J, Roukema J, Den Oudsten B. Sexual (dys) function and the quality of sexual life in patients with colorectal cancer: a systematic review. Annals of Oncology. 2011;23(1):19-27.

39. Kopp I, Bauhofer A, Koller M. Understanding quality of life in patients with colorectal cancer: comparison of data from a randomised controlled trial, a population based cohort study and the norm reference population. Inflammation Research. 2004;53(2):S130-S5.

40. Phipps E, Braitman LE, Stites S, Leighton JC. Quality of life and symptom attribution in long-term colon cancer survivors. Journal of evaluation in clinical practice. 2008;14(2):254-8.

41. Ramsey SD, Berry K, Moinpour C, Giedzinska A, Andersen MR. Quality of life in long term survivors of colorectal cancer. The American journal of gastroenterology. 2002;97(5):1228-34.

42. Marquis P, Marrel A, Jambon B. Quality of life in patients with stomas: the Montreux Study. Ostomy/wound management. 2003;49(2):48-55.

43. Ko CY, Maggard M, Livingston EH. Evaluating health utility in patients with melanoma, breast cancer, colon cancer, and lung cancer: a nationwide, population-based assessment1. Journal of Surgical Research. 2003;114(1):1-5.

\section{(i) (2)}

This work is licensed under a Creative Commons AttributionNon Commercial 4.0 International License. 